\title{
Could inactivation of platelets be key to preventing thrombocytopenia in dengue virus infected patients?
}
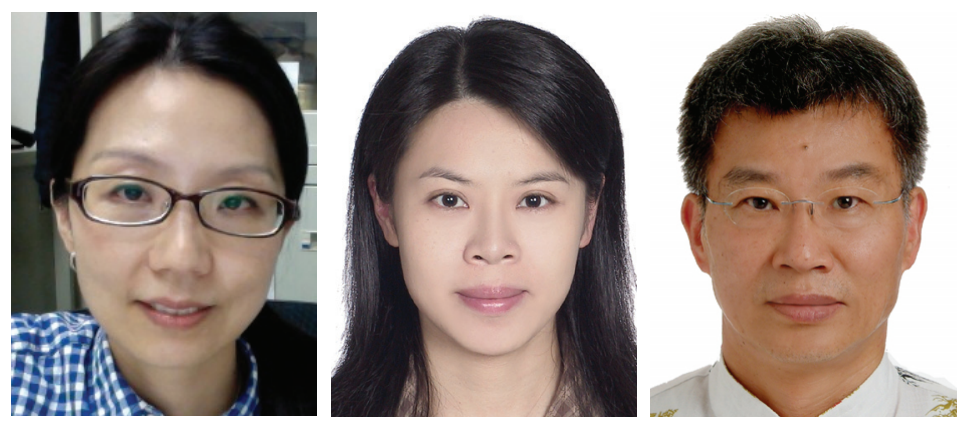

Yu-Chih Lo ${ }^{1,2}$, Yu-Wen Chien ${ }^{2,3}$ \& Guey Chuen Perng ${ }^{*, 2,4}$

First draft submitted: 13 April 2017; Accepted for publication: 20 April 2017; Published online: 12 July 2017

In recent years, dengue has been one of the most important mosquito-borne human viral diseases globally [1]. Although the disease is predominantly transmitted through the bite of Aedes mosquitoes carrying infectious dengue virus (DENV), recent evidence shows that other routes of introducing the virus to human beings are escalating and are likely to be underestimated [2]. Nevertheless, dynamic clinical presentations are the salient features in affected subjects, and numerous distinctive laboratory findings have been implicated in association with disease developments [3].

Wide clinical spectra of DENV infected people have been well documented, ranging from asymptomatic infection, mild dengue fever (DF) to potential life-threatening conditions, dengue hemorrhagic fever (DHF) and dengue shock syndrome (DSS), featured with plasma leakage likely from an increase in vascular permeability. In general, similar to many febrile diseases induced by viruses, dengue is a time-dependent febrile illness. However, people often do not recall or notice when they were bitten by mosquitoes, but roughly recall the time of fever onset. Cumulative data reveals that the majority of DENV infected individuals seek professional help on the fourth day after the fever starts [4]. By which time, according to the reported human studies, the time lapse is about 10 days after the DENV being inoculated into human bodies by the bite of mosquitoes. Coincidentally, this time likely corresponds to the beginning of clinical investigations. Hence, there are many biomarkers being proposed as candidates for differential diagnosis of DF and DHF/ DSS [5]. However, as of today, there are no consensus biomarkers available to differentiate DF from DHF. In addition, whether DHF is a consequence of the progression of DF remains to be further delineated

\section{KEYWORDS}

- dengue - flaviviruses

- mosquito-borne viral diseases

- therapeutic

- thrombocytopenia

'Department of Biotechnology \& Bioindustry Sciences, College of Bioscience \& Biotechnology, National Cheng Kung University, Tainan, Taiwan

${ }^{2}$ Institute of Basic Medical Sciences, College of Medicine, National Cheng Kung University, Tainan, Taiwan

${ }^{3}$ Department of Public Health, College of Medicine, National Cheng Kung University, Tainan, Taiwan

${ }^{4}$ Department of Microbiology \& Immunology, College of Medicine, National Cheng Kung University, Tainan, Taiwan

*Author for correspondence: gperng@mail.ncku.edu.tw

Future
Medicine 
“...the functionality of platelets during the febrile stage has been investigated and reveals that platelet dysfunction in the acute febrile stage are common." and clarified. Despite there being many different hypotheses on the mechanisms of how DHF develops, the true etiological factors have not been identified.

Human platelets are small, anucleate, colorless blood cells, $2-4 \mu \mathrm{m}$ in diameter containing granules, mitochondria and endoplasmic reticulum. Platelets are made in human bone marrow and travel through blood vessels to form clots to stop any bleeding that may happen if a blood vessel becomes damaged. Although the major role of platelets is maintaining hemostasis, they also play a significant role in many pathophysiologic processes, including thrombosis, hemorrhage, inflammation, pathogen host defense, tumor growth and metastasis [6]. In healthy human beings, the normal count of circulating platelets is approximately $150,000-450,000$ platelets/ $\mu \mathrm{l}$ of blood and the turnover rate on average is $7-10$ days. In general, when the platelet count drops to below 150,000 platelets/ $\mu \mathrm{l}$ in circulation, it is considered lower than normal and can be categorized as being a low platelet count, or so-called thrombocytopenia [6], and if a platelet count is below 50,000 platelets $/ \mu \mathrm{l}$, which is a potential life threatening event, caused by internal bleeding, an emergency treatment may be required.

Hematological disorder is one of the most common findings in dengue patients [7], and yet its significant role, both biologically and clinically, in the pathogenesis of DHF/DSS remains largely unexplored. Thrombocytopenia is a salient laboratory finding in both DF and DHF patients [1]. Significantly, it has been shown that platelet counts correlate to the degree of severity of the disease development [8] and the nadir platelet count has been corresponded to the most critical period in dengue subjects [1]. As such, investigations on the mechanisms leading to low platelet counts in dengue patients has been the main focus for many years. As the platelet count in dengue patients are time sensitive events, the fever onset day primarily relies upon the recall of infected subjects; the dynamic change of platelet counts in infected individuals is the most challenging task to be tabulated. In addition, other underlying diseases or medicines taken by the affected individuals may contribute to the low platelet counts in patients. Nevertheless, two major mechanisms contributing to the thrombocytopenia have been proposed: impaired production and platelet destruction with or without dysfunction [9]. These result in several different scenarios, including direct infection of platelets by DENV, suppression of megakaryopoiesis in bone marrow, reduction of megakaryocytes in bone marrow, inhibition of thrombopoiesis, shortening the life span, apoptosis, complement-mediated destruction and antibody-mediated phagocytosis by phagocytic cells $[7,8,10-12]$.

There are at least three distinctive stages of the clinical course in patients with DHF/DSS: febrile, critical and convalescent. The progression of the platelet counts is the following; at normal range during the early febrile stage, at the lowest in the critical phase and rebounded to normal range during the convalescent period [7]. Importantly, the status of platelets is activated during the febrile stage, which has recently been demonstrated to be correlated with the severity of thrombocytopenia in dengue patients [8]. Moreover, the functionality of platelets during the febrile stage has been investigated and reveals that platelet dysfunction in the acute febrile stage are common [7]. Furthermore, parameters derived from platelets contributing to the vascular permeability have been reported recently [5]. This cumulative evidence indicates that activated platelets play a significant role in DHF/ DSS. Researchers have, therefore, initiated the concept that therapeutic measures by inactivation of platelets might benefit dengue patients $[8,13]$.

Biologically, inactivation of platelets may benefit the affected dengue patients by mitigating the progression to severe dengue. However, it has been demonstrated that platelet count, occasionally, does not correlate with the bleeding tendency in dengue patients [7], suggesting alternative factors may contribute to the DHF/ DSS. Recent evidence suggests that platelets are part of the human immune system since the contents released in their active form play a role in modulating of the proper immune response in human hosts [14]. Interestingly, although abnormal immune response in dengue-infected subjects has been known for many decades, the causative mechanisms remain at large. This raises a scenario in which there are at least two additional and distinctive emerging functions of the platelets in dengue patients: mediating proper immune response in the host and assisting on the integrity of endothelial cells lining the blood vessel walls [15].

Scientifically, recent evidence demonstrates that platelets and their progenitor cells are likely to be infected by DENV in patients [8,16-19], suggesting that the timing of inactivated platelets occurs very early. Moreover, multifactorial factors engaging in the hemostasis of blood components and integrity, as well as functionality of endothelial cells, in dengue patients have been 
well documented [5]. As such, therapeutic measures to inactivate platelets in dengue patients, to some extent, may have some benefits [20]. As a whole, in order to find a precise countermeasure to mitigate or improve the progression of clinical conditions, the identification of etiologic mechanisms leading to low platelet counts, in affected dengue subjects, should be further delineated.

Financial \& competing interests disclosure

This work was, in part, supported by the Ministry of Health and Welfare (MOHW104-CDC-C-114-114901) and Ministry of Education, Taiwan. The Aim for the Top University Project to the National Cheng Kung University (NCKU), and Ministry of Science and Technology Grant (MOST 103-2320-B-006-030-MY3) (GC Perng) and Grant (MOST 104-2320-B-006-033-MY3) (Y-C Lo). The authors have no other relevant affliations or financial involvement with any organization or entity with a financial interest in or financial conflict with the subject matter or materials discussed in the manuscript apart from those disclosed.

No writing assistance was utilized in the production of this manuscript.

\section{References}

1 WHO. Global strategy for dengue prevention and control 2012-2020. WHO Press, Geneva, Switzerland (2012).

2 Perng G. Dengue human challenge model: is there a risk caveat? Persistent infection of dengue virus. J. Virol. Emerg. Dis (2015). www.sciforschenonline.org

3 Halstead SB. Predictors of dengue severity. J. Pediatr. 92(5), 429-431 (2016).

4 Tsai J-J, Liu L-T, Chang K et al. The importance of hematopoietic progenitor cells in dengue. Ther. Adv. Hematol. 3(1), 59-71 (2012).

5 John DV, Lin YS, Perng GC. Biomarkers of severe dengue disease - a review. J. Biomed. Sci. 22, 83 (2015)

6 Michelson AD. Platelet Biology. In: Platelets (2nd Edition). A Michelson (Eds). Academic Press, Elsevier, CA, USA (2007).

7 Srichaikul T, Nimmannitya S. Haematology in dengue and dengue haemorrhagic fever. Baillieres Best Pract. Res. Clin. Haematol. 13(2), 261-276 (2000).

8 Ojha A, Nandi D, Batra H et al. Platelet activation determines the severity of thrombocytopenia in dengue infection. Sci. Rep. 7, 41697 (2017).
9 Mitrakul C, Poshyachinda M, Futrakul P, Sangkawibha N, Ahandrik S. Hemostatic and platelet kinetic studies in dengue hemorrhagic fever. Am. J. Trop. Med. Hyg. 26(5 Pt 1), 975-984 (1977).

10 Tsai JJ, Chang K, Chen PC et al. Transient monocytosis subjugates low platelet count in adult dengue patients. Biomed. Hub 2:457785(2017).

11 Honda S, Saito M, Dimaano EM et al. Increased phagocytosis of platelets from patients with secondary dengue virus infection by human macrophages. Am. J. Trop. Med. Hyg. 80(5), 841-845 (2009).

12 Hottz ED, Medeiros-de-Moraes IM, Vieira-de-Abreu A et al. Platelet activation and apoptosis modulate monocyte inflammatory responses in dengue. J. Immunol. 193(4), 1864-1872 (2014).

13 Michels M, Alisjahbana B, De Groot PG et al. Platelet function alterations in dengue are associated with plasma leakage. Thromb. Haemost. 112(2), 352-362 (2014).

14 Elzey BD, Tian J, Jensen RJ et al. Plateletmediated modulation of adaptive immunity. A communication link between innate and adaptive immune compartments. Immunity 19(1), 9-19 (2003).
15 Nascimento EJ, Hottz ED, Garcia-Bates TM, Bozza F, Marques ET Jr, Barratt-Boyes SM. Emerging concepts in dengue pathogenesis: interplay between plasmablasts, platelets, and complement in triggering vasculopathy. Crit. Rev. Immunol. 34(3), 227-240 (2014).

16 Clark KB, Noisakran S, Onlamoon N et al. Multiploid $\mathrm{CD} 1^{+}$cells are the pre-dominant cell lineage infected during acute dengue virus infection in bone marrow. PLoS ONE 7(12), e52902 (2012).

17 Hsu AY, Wu SR, Tsai JJ et al. Infectious dengue vesicles derived from $\mathrm{CD} 61^{+}$cells in acute patient plasma exhibited a diaphanous appearance. Sci. Rep. 5, 17990 (2015).

18 Simon AY, Sutherland MR, Pryzdial EL. Dengue virus binding and replication by platelets. Blood 126(3), 378-385 (2015).

19 Noisakran S, Onlamoon N, Pattanapanyasat $\mathrm{K}$ et al. Role of $\mathrm{CD} 61^{(+)}$cells in thrombocytopenia of dengue patients. Int. J. Hematol. 96(5), 600-610(2012).

20 Lye DC, Lee VJ, Sun Y, Leo YS. Lack of efficacy of prophylactic platelet transfusion for severe thrombocytopenia in adults with acute uncomplicated dengue infection. Clin. Infect. Dis. 48(9), 1262-1265 (2009). 